\title{
Knowledge of healthcare professionals about poliomyelitis and postpoliomyelitis: a cross-sectional study
}

\author{
Claudio Andre Barbosa de Lira', Douglas Assis Teles Santos", Ricardo Borges Viana'"', Juliana Moreira Guimarães"', \\ Jéssica Nathalia Soares Oliveirav", Bolivar Saldanha Sousav", Marcos Gonçalves de Santana ${ }^{\mathrm{VII}}$, Rodrigo Luiz Vancini" ${ }^{\mathrm{VII}}$, \\ Marília Santos Andrade ${ }^{\mid \mathrm{x}}$, Pantelis Nikolaidis ${ }^{\mathrm{x}}$, Thomas Rosemann ${ }^{\mathrm{xI}}$, Beat Knechtle ${ }^{\mathrm{XII}}$
}

Universidade Federal de Goiás (UFG), Goiânia (GO), Brazil

'BSc, PhD. Associate Professor, Faculdade de Educação Física e Dança (FEFD), Universidade Federal de Goiás (UFG), Goiânia (GO), Brazil.

(D) https://orcid.org/0000-0001-5749-6877

"BSC, MSc. Assistent Professor, Colegiado de Educação Física,

Universidade do Estado da Bahia (UNEB), Teixeira de Freitas (BA), Brazil.

(D) https://orcid.org/0000-0002-7664-5468

"'BSC, PhD. Professor, Escola Superior de Educação Física e

Fisioterapia do Estado de Goiás (ESEFFEGO), Universidade Estadual de Goiás (UEG), Goiânia (GO), Brazil.

(iD https://orcid.org/0000-0001-9200-3185

"BSc. Nurse, Unidade Acadêmica Especial de Ciências da Saúde,

Universidade Federal de Jataí (UFJ), Jataí (GO), Brazil.

(D) https://orcid.org/0000-0002-6729-0335

`BSc. Biomedic, Unidade Acadêmica Especial de Ciências da Saúde

(UA-CISAU), Universidade Federal de Jataí (UFJ), Jataí (GO), Brazil.

(D) https://orcid.org/0000-0002-9531-4277

"MD, PhD. Physician, Hospital Israelita Albert Einstein, São Paulo-

Brazil, São Paulo (SP), Brazil.

(iD https://orcid.org/0000-0001-9682-5987

"WiBSc, PhD. Associate Professor, Unidade Acadêmica Especial de

Ciências da Saúde (UA-CISAU), Universidade Federal de Jataí (UFJ), Jataí (GO), Brazil.

(iD) https://orcid.org/0000-0001-7674-3263

wlBSC, PhD. Adjunct Professor, Centro de Educação Física e Desportos, Universidade Federal do Espirito Santo (UFES), Vitória (ES), Brazil.

(D) https://orcid.org/0000-0003-1981-1092

xphD. Physical Therapist and Associate Professor, Department of

Physiology, Universidade Federal de São Paulo (USP), São Paulo

(SP), Brazil.

(iD https://orcid.org/0000-0002-7004-4565

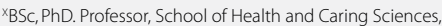

University of West Attica, Athens, Greece.

(D) https://orcid.org/0000-0001-8030-7122

x'MD, PhD. Physician and Professor, Institute of Primary Care,

University of Zurich, Zurich, Switzerland

(D) https://orcid.org/0000-0002-6436-6306

XIIMD, PhD. Physician and Scientific Assistant, Institute of Primary Care, University of Zurich, Zurich, Switzerland; and Researcher,

Medbase St. Gallen Am Vadianplatz, St. Gallen, Switzerland

(D) https://orcid.org/0000-0002-2412-9103

KEYWORDS (MeSH terms):

Poliomyelitis.

Physicians.

Nervous system.

Postpoliomyelitis syndrome.

Knowledge.

AUTHORS' KEY WORDS:

Medical education.

Neuromuscular disease

Late effects of polio.

\section{ABSTRACT}

BACKGROUND: Postpoliomyelitis syndrome is a clinical condition that can affect poliomyelitis survivors. OBJECTIVE: Our aim was to evaluate knowledge of poliomyelitis and postpoliomyelitis syndrome among Brazilian healthcare professionals.

DESIGN AND SETTING: Cross-sectional study conducted at a Brazilian public higher education institution located in the state of Goiás.

METHODS: The participants $(n=578$ ) were Brazilian physicians, physical therapists, nurses, nutritionists and psychologists. A self-administered questionnaire (30 questions) was designed to probe knowledge about poliomyelitis and postpoliomyelitis syndrome. From the questionnaire, we created a structured test to objectively evaluate the knowledge of these professionals. The test was composed of 20 questions and was scored over a range from 0 (totally ill-informed) to 20 (totally well-informed).

RESULTS: In general, the physicians, physical therapists and nurses demonstrated better understanding of poliomyelitis and postpoliomyelitis syndrome. The healthcare professionals who had received previous information about poliomyelitis and postpoliomyelitis syndrome had significantly higher scores than those who had never received information $(P<0.001)$. On average, this difference was approximately $28.6 \%$. CONCLUSIONS: The findings from the present study indicate that there is a critical need for improvement of knowledge about postpoliomyelitis syndrome among Brazilian healthcare professionals. The services provided by these professionals may therefore become compromised. Furthermore, public healthcare initiatives should be implemented to improve knowledge among healthcare professionals.

\section{INTRODUCTION}

Poliomyelitis is an infectious viral disease that may attack people at any age. It affects the nervous system, resulting in paralysis and muscle spasms, and in some cases encephalitis. ${ }^{1,2}$ There are several regions in the world that are certified as free from poliomyelitis. ${ }^{3}$ However, there are some endemic countries, such as Afghanistan, Nigeria and Pakistan, and there are records of imported cases in some African countries. ${ }^{3}$ In Brazil, even though poliomyelitis has been eradicated, according to the Brazilian Health Ministry, 312 Brazilian municipalities have polio vaccine coverage of below $50 \%$. This creates a state of alert and threatens the eradication of the disease. Therefore, there is a need to maintain permanent and effective actions of disease surveillance and adequate levels of immunological protection for the population. ${ }^{4}$

Postpoliomyelitis syndrome (PPS) is the term used to describe a collection of signs and symptoms that may be experienced by individuals afflicted by paralytic poliomyelitis after years of clinical and functional stability. ${ }^{5}$ The signs and symptoms that characterize PPS are new muscle weakness, muscle fatigue, muscle atrophy, muscle and joint pain, sleep disturbances, intolerance to cold, respiratory and swallowing difficulties and a recent increase in body mass. ${ }^{6-8}$ It is a slowly progressive disease, usually insidious, with subacute onset, sometimes resulting in significant restrictions of activities associated with everyday life. ${ }^{7,9-16}$

Although the pathophysiology of PPS is unclear, different mechanisms have been proposed. The most accepted mechanism postulates that degeneration or dysfunction of giant motor units, manifested by peripheral deterioration (axon and/or neuromuscular junction), probably as a result of metabolic requirements of giant motor units (muscle overuse), has a central role in the etiology of the disease. ${ }^{9,17}$ However, there are different hypotheses associated with the pathophysiology of 
the disease, which include: muscle disuse, the normal loss of motor units with age, predisposition to motor neuron degeneration due to glial vascular and lymphatic damage, reactivation of the virus or persistent infection, immunological factors related to poliomyelitis, ${ }^{9,15,18-20}$ the effect of growth hormone and the combined effect of overuse, disuse, pain, body mass gain or other diseases. ${ }^{9,18,20}$

Regarding the diagnostic criteria for PPS, a clinical approach aimed at ruling out other neurological diseases, orthopedic conditions, psychiatric disorders or even consequences linked to the aging process is required, since these conditions could develop the same signs and symptoms as seen in PPS. ${ }^{9,21}$ Therefore, it is extremely important that patients should be managed by a multidisciplinary healthcare professional team that includes neurologists, rheumatologists, orthopedists, pulmonologists, physical education professionals, physical therapists, nutritionists, nurses and psychologists. ${ }^{9,22,23}$

Despite the significant decline in the incidence of paralytic poliomyelitis, ${ }^{3}$ PPS will remain a major health problem for many years. In western countries, where the last large epidemics date back to the 1940s and 1950s, many survivors of paralytic poliomyelitis are now aged between 70 and 80 years. ${ }^{24}$ In Brazil, the last major outbreak was in $1984 .{ }^{24}$ Therefore, the future outlook is for continued or even increased need for rehabilitation programs and management of people with PPS. ${ }^{9,25}$ In addition, according to the World Health Organization, it is estimated that there are about 18 million people alive who were affected by paralytic poliomyelitis. ${ }^{3}$

As it is reasonable to assume that success in the treatment of any disease depends on the knowledge that the healthcare professional has about the disease, assessment of knowledge about poliomyelitis and PPS among healthcare professionals is of great value. Previously, it was demonstrated that physical education professionals have misconceptions about poliomyelitis and PPS. ${ }^{8}$ Therefore, it is reasonable to assume that other healthcare professionals present similar misconceptions regarding poliomyelitis and PPS. Furthermore, other studies have shown that healthcare professionals can present misconceptions about epilepsy, ${ }^{26,27}$ acquired immune deficiency syndrome (AIDS) ${ }^{28}$ cancer ${ }^{29}$ hypertension and diabetes. ${ }^{30}$

\section{OBJECTIVE}

The aim of this study was to verify the knowledge of health professionals about poliomyelitis and PPS regarding the pathophysiology, etiology, symptomatology and forms of treatment of PPS.

\section{METHODS}

\section{Participants}

This was a cross-sectional study in which a total of 578 participants (454 women and 124 men) were recruited. This was done using different sources of advertisement (i.e. internet, local newspapers, magazines and billboards in universities, clinics, hospitals and gyms). The inclusion criterion for the study was that the participants should be professionals with at least an undergraduate degree in medicine, physical therapy, nursing, nutrition or psychology. Healthcare professionals with specialization in neurology and/or neuromuscular disorders were excluded from the study.

All the participants were informed of the aim of the study and experimental procedures, and written informed consent was obtained from each participant before any data were collected. All procedures involved in this study were approved by the Universidade Federal de Goiás (UFG) Ethics Committee (protocol number: 198/2009; approved on August 1,2010) and followed the principles outlined in the Declaration of Helsinki.

\section{Questionnaire}

To evaluate knowledge about poliomyelitis and PPS, we used the same questionnaire as described by de Lira et al. ${ }^{8}$ This was a selfadministered questionnaire divided into three parts: (i) personal data; (ii) knowledge about poliomyelitis; and (iii) knowledge about PPS. The questionnaire consisted of 14 questions about poliomyelitis and 16 questions about PPS and the aspects of these conditions that were addressed related to pathophysiology, diagnosis, forms of treatment, prognosis and previous work experience, with simple scales for closed-type responses. This questionnaire had been prepared in accordance with previous recommendations. ${ }^{31,32}$ The questions are shown in Tables 2 and 3.

In addition, a knowledge assessment test was created. This aimed to objectively show the knowledge of the professionals interviewed. The test comprised 20 questions within the questionnaire, which were analyzed as a score for correct responses, ranging from 0 to 20 points. These questions are marked with an asterisk in Tables 2 and $\mathbf{3}$.

\section{Statistical analysis}

Descriptive statistics were used to analyze the findings (mean, standard deviation and absolute and relative frequencies). The Gaussian distribution of the sample was tested by means of the Kolmogorov-Smirnov test. One-way analysis of variance (ANOVA) was used to compare age and professional experience among categories of healthcare professionals. The chi-square test was used to determine the association between healthcare professionals and knowledge about poliomyelitis and PPS. The KruskalWallis test was used to compare scores from the questionnaire, obtained by the different healthcare professional categories, followed by Dunn post-hoc comparison. The Mann-Whitney test was used to compare scores obtained in the questionnaire between the exposure ('yes' and 'no') groups. For all statistical procedures, the significance level assumed was $5 \%$. 


\section{RESULTS}

\section{Participants}

A total of 578 participants ( 454 women and 124 men, comprising a convenience sample) were evaluated. Of these, 69 were physicians (19 women and 50 men), 151 were physical therapists (116 women and 35 men), 224 were nurses (203 women and 21 men), 78 were nutritionists ( 74 women and 4 men) and 56 were psychologists (42 women and 14 men). Out of the 578 participants approached, $305(52.8 \%)$ had a specialization degree, $126(21.8 \%)$ had a $\mathrm{PhD}, 86$ (14.9\%) had a master's qualification, 59 (10.2\%) only had an undergraduate degree and two $(0.2 \%)$ did not report their highest graduation level. The other characteristics of the participants are presented in Table 1.

\section{Knowledge about poliomyelitis}

The first part of the questionnaire was designed to assess knowledge about poliomyelitis. Out of the 578 participants approached, $576(99.7 \%)$ had heard about poliomyelitis. Surprisingly, two nurses $(0.35 \%$ of the participants) had never heard of poliomyelitis (Table 2). The chi-square test did not reveal any significant association between healthcare professional category and having heard about poliomyelitis $(\mathrm{P}=0.530)$.

Out of the 578 participants approached, 461 (79.8\%) answered that they had received information about the disease through books, pamphlets and lectures. While approximately $90 \%$ of the physicians, physical therapists and nurses had had access to information on how to deal with poliomyelitis in their undergraduate courses, this proportion fell to approximately half among the nutritionists (43.6\%) and psychologists (50.0\%) (Table 2). The chi-square test revealed that there was a significant association between healthcare professional category and receiving information about poliomyelitis $(\mathrm{P}<0.001)$.

With regard to the biological agent that causes poliomyelitis, some healthcare professionals reported that they did not know that the disease is caused by a virus (7.6\%); and $4.0 \%$ of the participants stated that poliomyelitis is not caused by a virus. More than $90 \%$ of the physicians, physical therapists and nurses and approximately $70 \%$ of the nutritionists and psychologists correctly answered that poliomyelitis is caused by a virus. The chi-square test revealed that there was a significant association between healthcare professional category and knowledge of the biological agent that causes poliomyelitis $(\mathrm{P}<0.001)$.
Out of the 578 participants approached, $78.3 \%$ of the physicians, $39.7 \%$ of the physical therapists, $54.5 \%$ of the nurses, $21.8 \%$ of the nutritionists and $25 \%$ of the psychologists knew that poliomyelitis can be spread through water and/or food contaminated with feces from a sick person. The chi-square test revealed that there was a significant association between healthcare professional category and knowing that poliomyelitis can be spread through water and/ or food contaminated with feces from a sick person $(\mathrm{P}<0.001)$.

Regarding the symptoms of poliomyelitis, $72.5 \%$ of the physicians, $41.1 \%$ of the physical therapists, $48.7 \%$ of the nurses, $26.9 \%$ of the nutritionists and $26.8 \%$ of the psychologists knew that poliomyelitis can cause gastrointestinal symptoms. The chi-square test revealed that there was a significant association between healthcare professional category and knowing that poliomyelitis can cause gastrointestinal symptoms $(\mathrm{P}<0.001)$.

Regarding neuromuscular symptoms, more than $90 \%$ of the healthcare professionals knew that poliomyelitis can cause neuromuscular symptoms such as paralysis, paresis, muscle atrophy and weakness. The chi-square test did not reveal any significant association between healthcare professional category and knowing that poliomyelitis can cause neuromuscular symptoms $(\mathrm{P}=0.262)$.

In relation to recovery of functional capacity after the acute poliomyelitis stage, $82.6 \%$ of the physicians, $70.2 \%$ of the physical therapists, $54 \%$ of the nurses, $33.3 \%$ of the nutritionists and $48.2 \%$ of the psychologists knew that after the acute poliomyelitis stage, patients can recover the functional capacity of affected structures. The chi-square test revealed that there was a significant association between healthcare professional category and knowing that patients can recover the functional capacity of affected structures $(\mathrm{P}<0.001)$.

Regarding epidemiology, between 60 and $80 \%$ of the healthcare professionals knew that poliomyelitis is a disease that has not been eradicated worldwide. The chi-square test did not reveal any significant association between healthcare professional categories and knowing that poliomyelitis is a disease that has not been eradicated around the world $(\mathrm{P}=0.406)$. Also, there was no significant association between health professional category with regard to knowing that a poliomyelitis vaccine is available $(\mathrm{P}=0.133)$. Indeed, more than $90 \%$ of the healthcare professionals knew that a vaccine is available to prevent poliomyelitis.

With regard to treatment, $94.2 \%$ of the physicians, $78.8 \%$ of the physical therapists, $73.7 \%$ of the nurses, $33.3 \%$ of the nutritionists

Table 1. Characteristics of participants $(n=578)$

\begin{tabular}{lccccc} 
Variable (years) & Physicians $n=69$ & Physical therapists $n=151$ & Nurses $n=224$ & Nutritionists $n=78$ & Psychologists $n=56$ \\
Age & $42.0 \pm 13.4$ & $32.1 \pm 8.3^{*}$ & $33.7 \pm 14.0^{*}$ & $30.3 \pm 8.0^{*+}$ & $36.1 \pm 11.5^{*}$ \\
Professional experience & $17.8 \pm 12.6$ & $8.2 \pm 8.0^{*}$ & $7.9 \pm 8.8^{*}$ & $7.3 \pm 9.1^{*}$ & $11.2 \pm 9.3^{*}$ \\
\hline
\end{tabular}

Data are presented as mean \pm standard deviation; *Statistically different from physicians (one-way analysis of variance (ANOVA) and Tukey post-test; $P<0.05$ );

'Statistically different from psychologists (one-way ANOVA and Tukey post-test; $\mathrm{P}<0.05$ ). 
Table 2. Answers among healthcare professionals relating to poliomyelitis

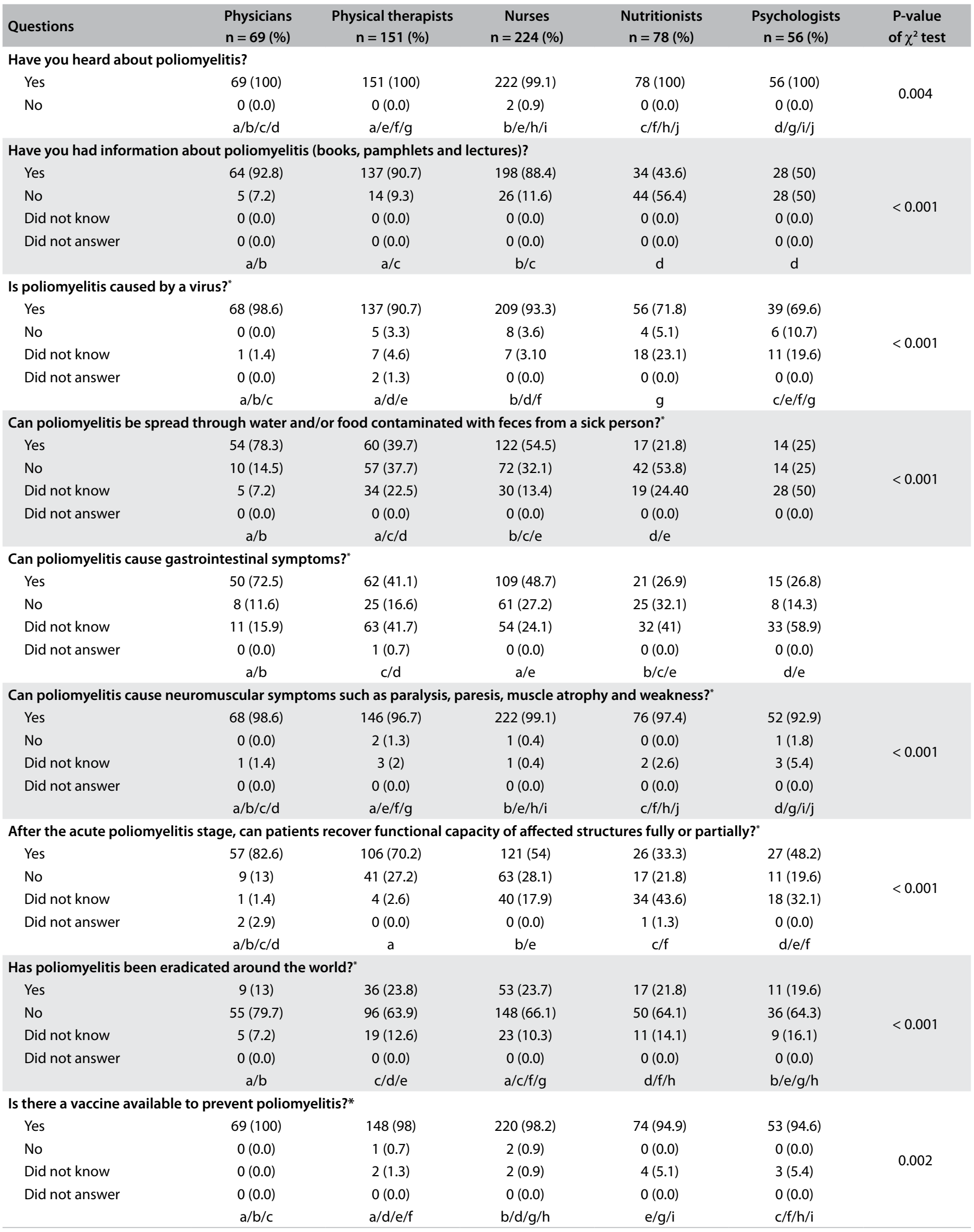


Table 2. Continuation.

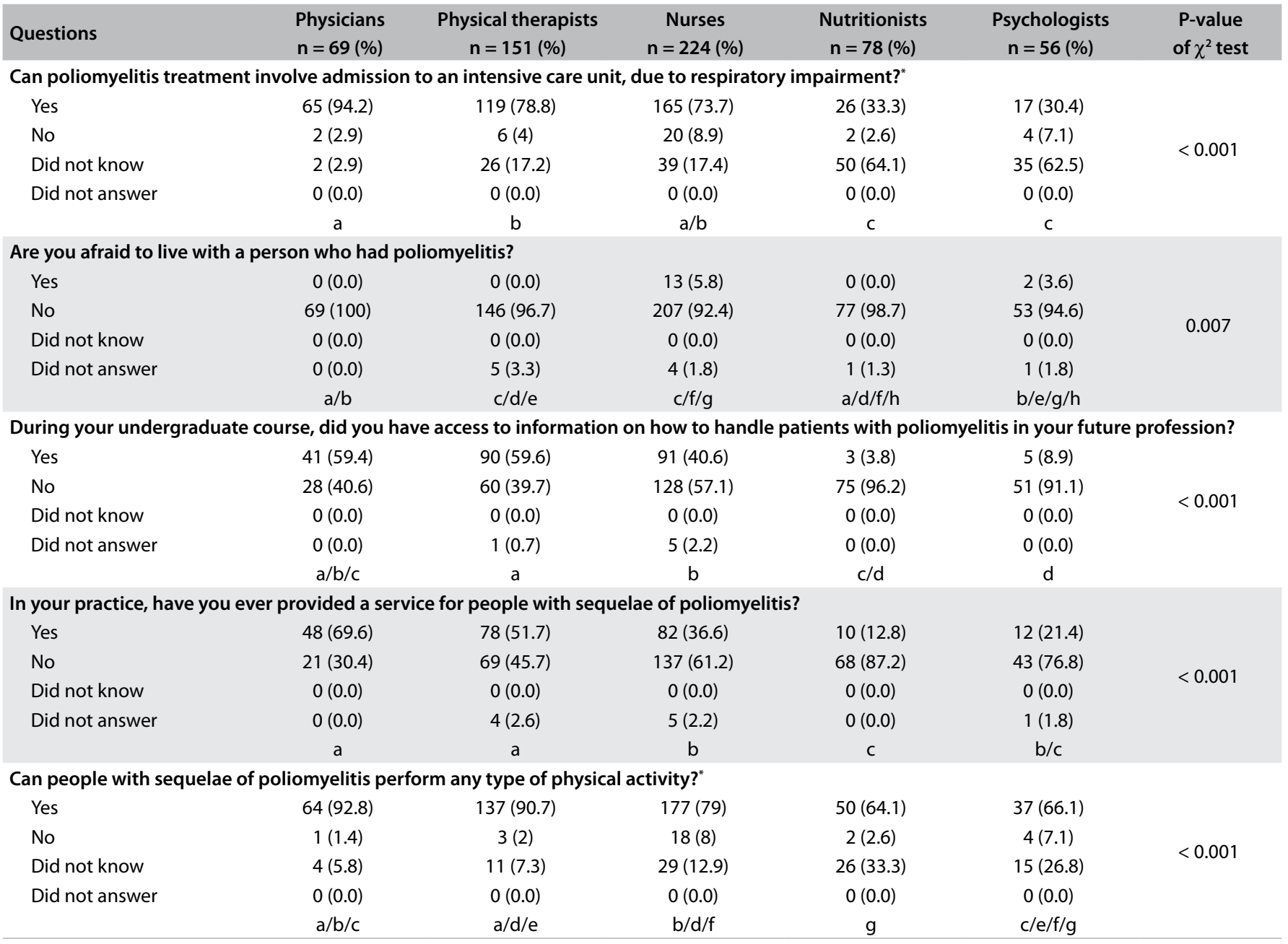

"Question that composed the knowledge assessment test on paralytic poliomyelitis and PPS. Frequencies followed by the same letters, in the rows, did not differ.

and $30.4 \%$ of the psychologists knew that poliomyelitis treatment can involve admission to an intensive care unit, due to respiratory impairment. The chi-square test revealed that there was a significant association between healthcare professional categories and knowing that poliomyelitis treatment can involve admission to an intensive care unit, due to respiratory impairment $(\mathrm{P}<0.001)$.

Surprisingly, 15 healthcare professionals (2.6\%) said that they were afraid to live with people with poliomyelitis (Table 2). The chisquare test revealed that there was a significant association between healthcare professional category and being afraid to live with people with poliomyelitis $(\mathrm{P}=0.001)$.

Only $39.8 \%$ had received information about how to deal with patients with poliomyelitis during their undergraduate courses. Specifically, $59.5 \%$ of the physicians, $59.6 \%$ of the physical therapists, $40.6 \%$ of the nurses, $3.8 \%$ of the nutritionists and $8.9 \%$ of the psychologists had received information about poliomyelitis during their undergraduate courses. The chi-square test revealed that there was a significant association between healthcare professional category and provision of information about poliomyelitis during undergraduate courses $(\mathrm{P}<0.001)$.

Regarding physical exercise, $92.8 \%$ of the physicians, $90.7 \%$ of the physical therapists, $79.0 \%$ of the nurses, $64.1 \%$ of the nutritionists and $66.1 \%$ of the psychologists responded that people with poliomyelitis sequelae can perform some kind of physical activity. The chi-square test revealed that there was a significant association between healthcare professional category and knowing about physical exercise $(\mathrm{P}<0.001)$.

\section{Knowledge about postpoliomyelitis syndrome}

The second part of the questionnaire was designed to assess knowledge about PPS. Out of the 578 participants approached, 243 (42\%) had heard about PPS (Table 3). Specifically, 60.9\% of the physicians, $67.5 \%$ of the physical therapists, $34.8 \%$ of the nurses, $12.8 \%$ of the nutritionists and $19.6 \%$ of the psychologists 
Table 3. Answers among healthcare professionals relating to post-poliomyelitis syndrome (PPS)

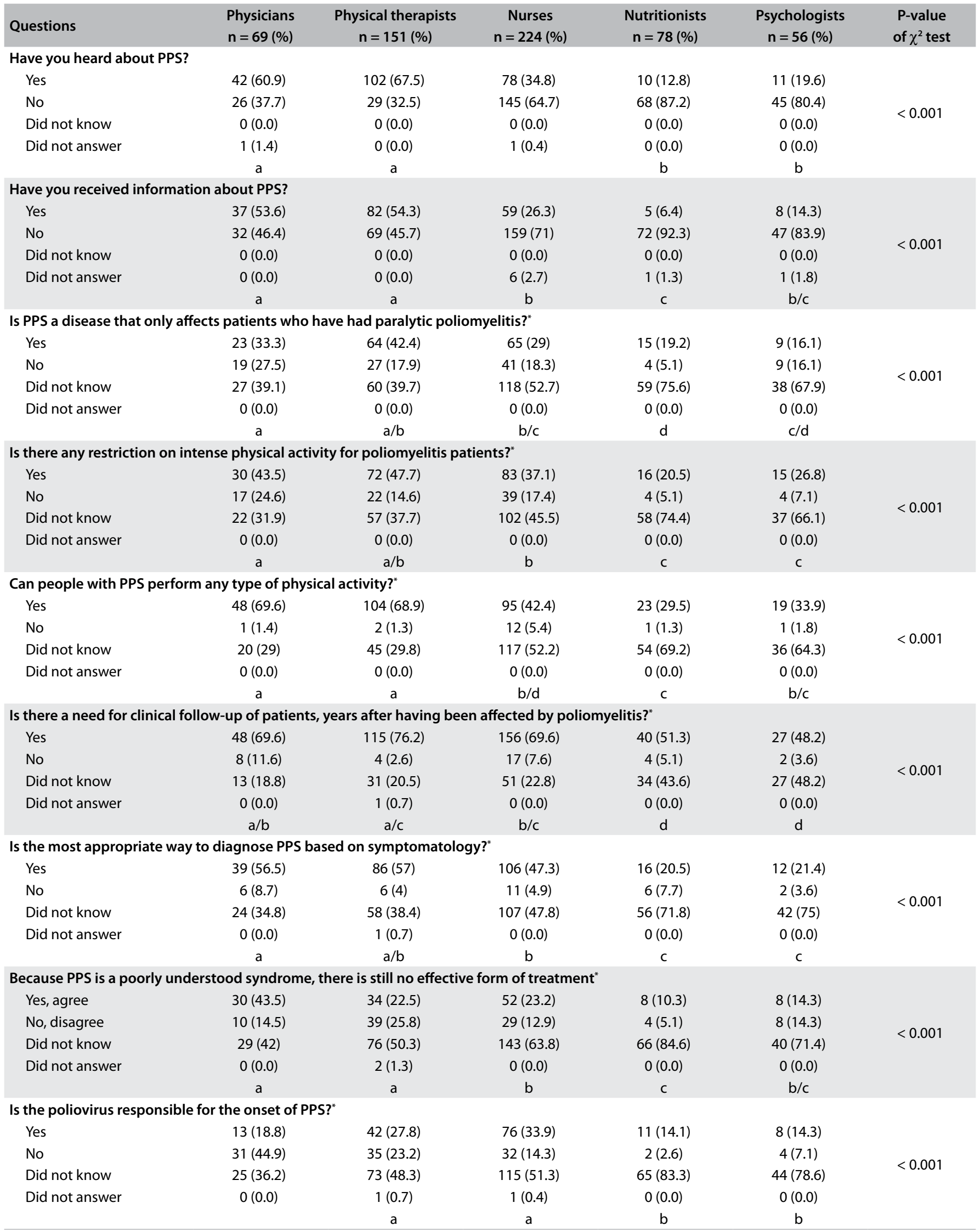


Table 3. Continuation.

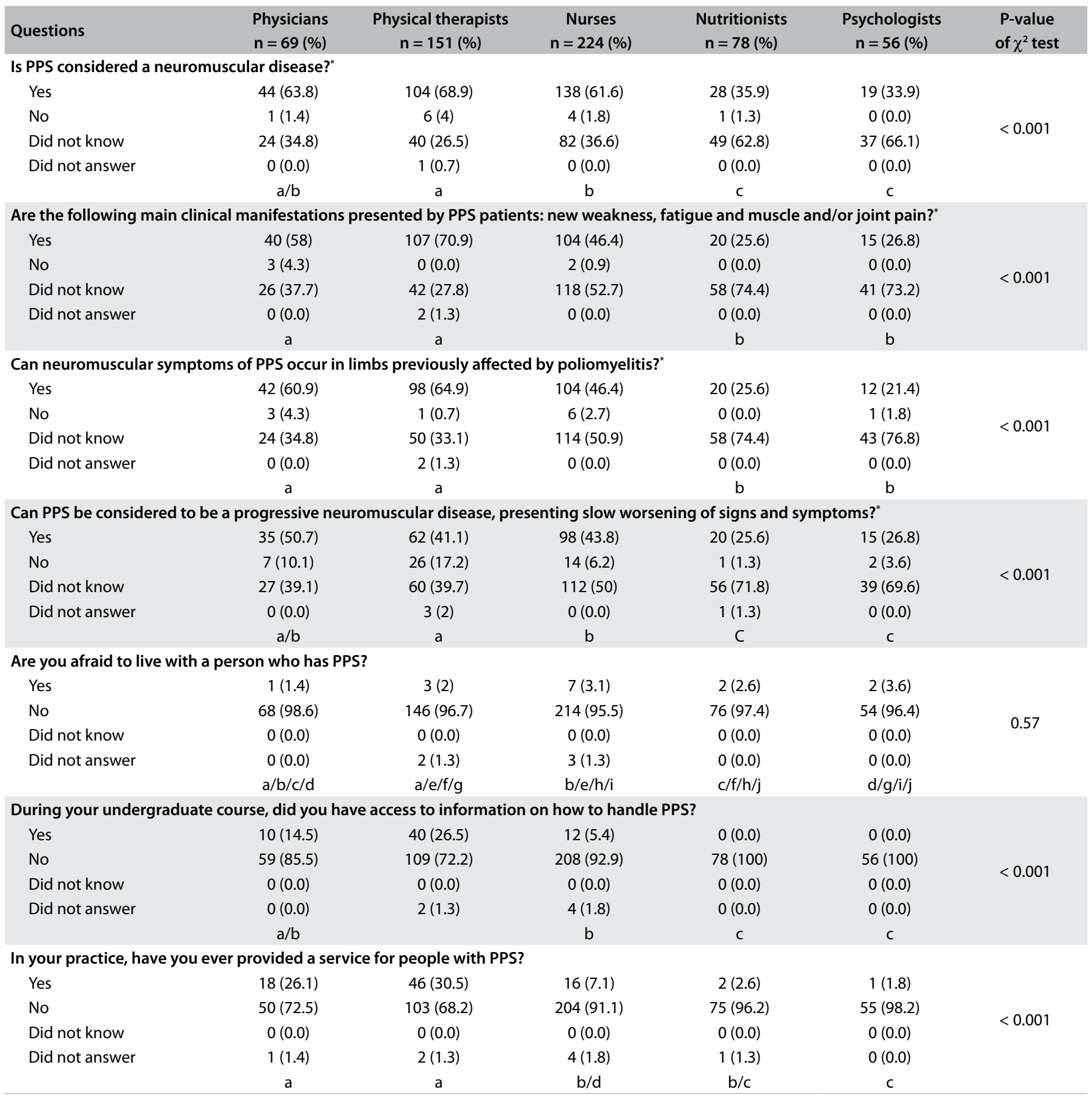

"Question that composed the knowledge assessment test on paralytic poliomyelitis and PPS. Frequencies followed by the same letters, in the rows, did not differ.

had heard about PPS. The chi-square test revealed that there was a significant association between healthcare professional category and having heard about PPS $(\mathrm{P}<0.001)$.

Out of the 578 participants approached, 373 (65.6\%) answered that they had received no information about PPS. While $53.6 \%$ of the physicians and $54.3 \%$ of the physical therapists had received information about PPS, only $26.3 \%$ of the nurses, $6.4 \%$ of the nutritionists and $14.3 \%$ of the psychologists had received information about PPS (Table 3). The chi-square test revealed that there was a significant association between healthcare professional category and having received information about PPS $(\mathrm{P}<0.001)$.

Regarding pathophysiology, 302 (52.2\%) did not know that the PPS affects only people who have had paralytic poliomyelitis in the past. While $33.3 \%$ of physicians and $42.4 \%$ of physical 
therapists knew that PPS is a disease that only affects patients who have had paralytic poliomyelitis, only $29 \%$ of the nurses, $19.2 \%$ of the nutritionists and $16.1 \%$ of the psychologists understood this. The chi-square test revealed that there was a significant association between healthcare professional category and knowing that PPS is a disease that only affects patients who have had paralytic poliomyelitis $(\mathrm{P}<0.001)$.

In relation to restrictions that the patient must obey, 276 (47.8\%) did not know whether there is any restriction on physical activity for people with poliomyelitis sequelae and only 216 (37.4\%) knew that exercise should be limited among people with paralytic poliomyelitis sequelae (especially intense exercise). Specifically, 31.9\% of the physicians, $37.7 \%$ of the physical therapists, $45.5 \%$ of the nurses, $74.4 \%$ of the nutritionists and $66.1 \%$ of the psychologists did not know about any restriction on intense physical activity for PPS patients. The chi-square test revealed that there was a significant association between healthcare professional category and knowing about any restriction on intense physical activity for PPS patients $(\mathrm{P}<0.001)$.

Furthermore, $50 \%$ of the healthcare professionals answered that PPS patients can perform any type of physical activity and only $2.9 \%$ of them correctly answered that PPS patients cannot perform any type of physical activity (Table 3 ). The chi-square test revealed that there was a significant association between healthcare professional category and knowing that PPS patients cannot perform any type of physical activity $(\mathrm{P}<0.001)$.

Regarding treatment, 386 (66.9\%) believed that clinical follow-up is necessary, even years after acute poliomyelitis, while 156 (27.0\%) did not know whether there is a need for clinical follow-up years after an individual has had polio. The chi-square test revealed that there was a significant association between healthcare professional category and knowing about the need for clinical follow-up of patients, years after having been affected by poliomyelitis $(\mathrm{P}<0.001)$.

Also regarding treatment, approximately $61.1 \%$ of the healthcare professionals did not know whether there is an effective treatment for PPS. Specifically, $42.0 \%$ of the physicians, $50.3 \%$ of the physical therapists, $63.8 \%$ of the nurses, $84.6 \%$ of the nutritionists and $71.4 \%$ of the psychologists did not know about this issue. The chi-square test revealed that there was a significant association between healthcare professional category and this matter $(\mathrm{P}<0.001)$.

With regard to diagnosis, 287 (49.7\%) did not know that the most appropriate way to diagnose PPS is based on symptomatology. Specifically, $34.8 \%$ of the physicians, $38.4 \%$ of the physical therapists, $47.8 \%$ of the nurses, $71.8 \%$ of the nutritionists and $75 \%$ of the psychologists did not know about this issue. The chi-square test revealed that there was a significant association between healthcare professional category and this matter $(\mathrm{P}<0.001)$.
Regarding PPS etiology, only 104 (18.0\%) knew that poliovirus is not responsible for PPS. Specifically, $44.9 \%$ of the physicians, $23.2 \%$ of the physical therapists, $14.3 \%$ of the nurses, $2.6 \%$ of the nutritionists and $7.1 \%$ of the psychologists did not know about this issue. The chi-square test revealed that there was a significant association between healthcare professional category and this matter $(\mathrm{P}<0.001)$.

Regarding PPS classification, 232 healthcare professionals (40.1\%) did not know that PPS is a neuromuscular disease. Specifically, $34.8 \%$ of the physicians, $26.5 \%$ of the physical therapists, $36.6 \%$ of the nurses, $62.8 \%$ of the nutritionists and $66.1 \%$ of the psychologists did not know about this issue. The chi-square test revealed that there was a significant association between healthcare professional category and this matter $(\mathrm{P}<0.001)$.

Regarding the main clinical manifestations presented by PPS patients, 285 of the healthcare professionals (49.3\%) did not know that the main clinical manifestations of PPS are new weakness, fatigue and muscle and/or joint pain. Specifically, while $58 \%$ of the physicians, $70.9 \%$ of the physical therapists and $46.4 \%$ of the nurses knew about this topic, only $25.6 \%$ of the nutritionists and $26.8 \%$ of the psychologists knew that PPS patients can present with new weakness, fatigue and muscle and/or joint pain. The chi-square test revealed that there was a significant association between healthcare professional category and this matter $(\mathrm{P}<0.001)$.

Furthermore, while $60.9 \%$ of the physicians, $64.9 \%$ of the physical therapists and $46.4 \%$ of the nurses knew that neuromuscular symptoms of PPS occur in limbs previously affected by poliomyelitis, only $25.6 \%$ of the nutritionists and $21.4 \%$ of the psychologists knew about this topic. The chi-square test revealed that there was a significant association between healthcare professional category and this matter $(P<0.001)$. A similar pattern was found for answers to question 27.

Surprisingly, 15 participants (2.6\%) said that they were afraid to live with people with PPS. The chi-square test did not reveal any association between healthcare professional category and being afraid to live with a person who has PPS $(\mathrm{P}=0.877)$.

Regarding the provision of information about this disease during the undergraduate course, only 62 participants (10.7\%) reported that during their undergraduate courses they had had access to information about management of PPS, and only 83 healthcare professionals (14.4\%) reported that they had already provided services to people with PPS (Table 3). The chi-square test did not reveal any association between healthcare professional category and these topics $(\mathrm{P}<0.001)$.

\section{Knowledge of the professionals about paralytic poliomyelitis and PPS}

With regard to the questionnaire that was created, the professionals scored on average $11.0 \pm 4.4$ (which corresponded to 
approximately $55 \%$ of the total score), out of a maximum score of 20. Specifically, the Kruskal-Wallis test $\left[\mathrm{X}^{2}(4)=107.500 ; \mathrm{P}<0.001\right]$ demonstrated that the knowledge of the physicians, physical therapists and nurses was significantly higher than that of the nutritionists and psychologists $(\mathrm{P}<0.05$, Figure 1); and that the knowledge of the physicians and physical therapists was significantly higher than that of the nurses, nutritionists and psychologists $(\mathrm{P}<0.05)$.

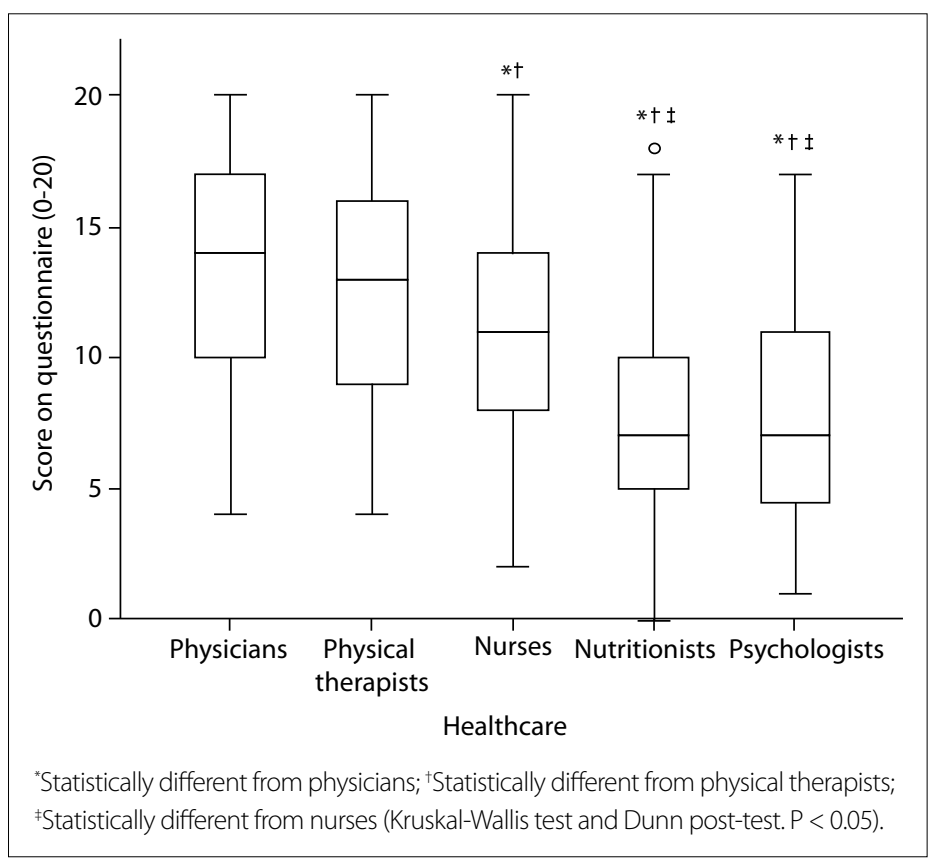

Figure 1. Questionnaire result (0-20) separated according to healthcare professional categories.

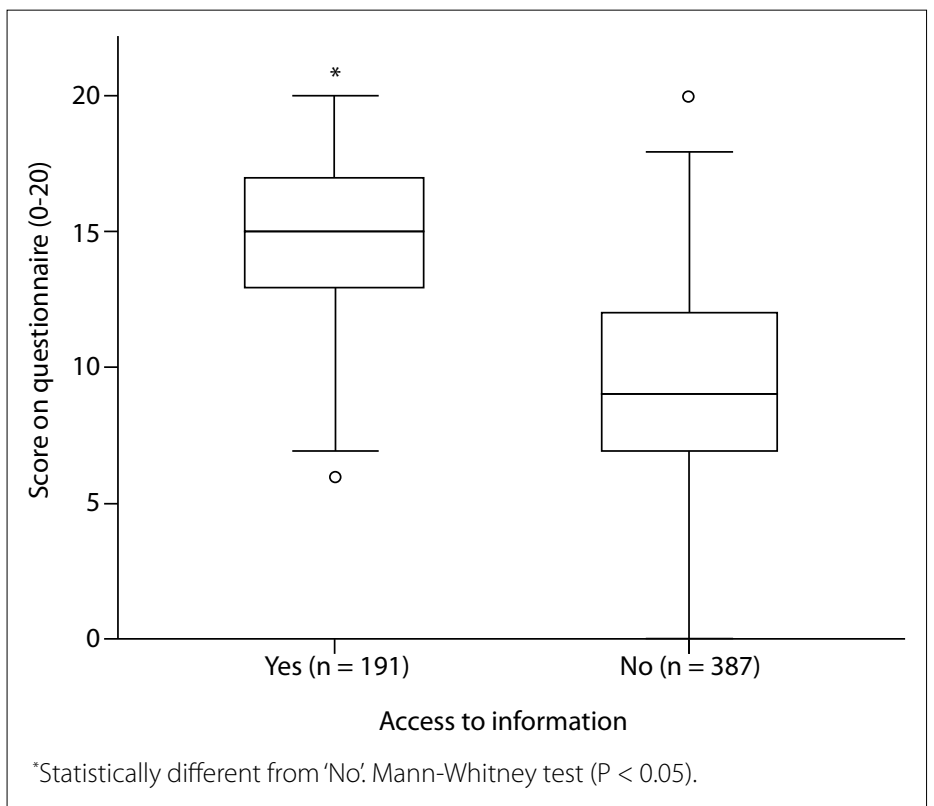

Figure 2. Questionnaire result (0-20) separated according to healthcare professionals with (Yes; $n=191$ ) or without (No; $n=387$ ) access to information about poliomyelitis and post-poliomyelitis syndrome.
We also found that healthcare professionals who had received previous information about poliomyelitis and PPS had significantly higher scores than those who had never received information $(\mathrm{P}<0.001)$. On average, this difference was approximately $28.6 \%$ (Figure 2). Five volunteers did not respond to this question.

\section{DISCUSSION}

This study evaluated knowledge about paralytic poliomyelitis and PPS among healthcare professionals (physicians, physical therapists, nurses, nutritionists and psychologists). Considering that success in treating a disease depends on the knowledge level that healthcare professionals have, with regard to etiology, signs and symptoms and management of the disease, studies with the aim of investigating the knowledge of these professionals about a particular disease are important. Despite finding misconceptions about poliomyelitis and PPS in all the healthcare professional categories assessed, our results revealed that physicians, physical therapists and nurses present higher knowledge about poliomyelitis and PPS than nutritionists and psychologists. Furthermore, we found that those who had received previous information about poliomyelitis and PPS had significantly higher scores than those who had never received information.

Previous studies have investigated healthcare professionals' knowledge about certain diseases. Morin et al. ${ }^{33}$ investigated physicians' knowledge about cryptosporidiosis and reported that these professionals did not have adequate knowledge about this disease. Vancini et al. ${ }^{26}$ investigated the knowledge of physical education professionals about epilepsy and also found low knowledge among these professionals. Regarding paralytic poliomyelitis and PPS, de Lira et $\mathrm{al}^{8}{ }^{8}$ found that physical education professionals had low knowledge about these diseases. Therefore, our results are in line with the literature.

The low level of knowledge about paralytic poliomyelitis and PPS can be explained because poliomyelitis is a disease that has been eradicated in various countries (including Brazil). ${ }^{34}$ In addition, PPS is a relatively unknown disease and physicians commonly confound the signs and symptoms of this disease with the aging process. Only recently, as a result of an initiative led by professionals at the Federal University of São Paulo, PPS was included in the International Classification of Diseases. ${ }^{35}$ Therefore, our findings were expected.

It is necessary to highlight that the low levels of knowledge demonstrated by these healthcare professionals (especially by the nutritionists and psychologists) are worrying, because it is reasonable to assume that patient care procedures can be influenced by the professional knowledge level. In this context, we found that healthcare professionals who had received previous information about poliomyelitis and PPS had significantly higher scores than those who had never received information. This result suggests 
that universities should include information about poliomyelitis and PPS in their undergraduate curricula, in order to improve the students' knowledge about poliomyelitis and, consequently, as future professionals. Not least, this result suggests that continuing education programs should be implemented as a government initiative. Furthermore, the low knowledge about paralytic poliomyelitis and PPS is worrying, because it is reasonable to assume that low knowledge could decrease professionals' ability to provide counseling about the importance of vaccines.

We also found that physicians, physical therapists and nurses presented higher knowledge than psychologists and nutritionists, as demonstrated by the scores in the knowledge assessment test. Considering that approximately $90 \%$ of the physicians, physical therapists and nurses had access to information on how to deal with poliomyelitis in their undergraduate studies and that this proportion fell to approximately $50 \%$ among the nutritionists and psychologists, this result was expected. Indeed, the fact that psychologists and nutritionists demonstrate less knowledge about the disease can be explained by the lack of material dedicated to infectious diseases in the undergraduate curricula of such courses. In particular, it is extremely important that physicians have high knowledge about the criteria for diagnosing PPS, because it is a syndrome for which the symptoms include new muscle weakness and muscle fatigue, among patients who have a history of paralytic poliomyelitis. ${ }^{22,25}$ However only $33 \%$ of the physicians knew that PPS affects individuals who have had polio in the past, and this is important because it is the main diagnostic criterion.

With regard to knowing that PPS is considered to be a progressive neuromuscular disease, with slow worsening of signs and symptoms, approximately $51 \%$ of the physicians had correct knowledge, while $42 \%$ of the physical therapists, $43 \%$ of the nurses, $26 \%$ of the nutritionists and $27 \%$ of the psychologists had correct knowledge. This shows that there are wide differences in knowledge among healthcare professionals. This result can probably be explained by the fact that physicians, physical therapists and nurses are directly involved in diagnosis and treatment in hospitals and clinics ${ }^{25}$ and, as mentioned above, by the presence of material dedicated to infectious diseases in the undergraduate curricula. On the other hand, the participation of nutritionists and psychologists is associated with ameliorating secondary symptoms, such as recent body mass gain ${ }^{36}$ and mood disorders. ${ }^{34}$

Although paralytic poliomyelitis has been eradicated in most countries (including Brazil), there are still some countries with new cases of paralytic poliomyelitis. ${ }^{37}$ In 2018, the World Health Organization (WHO) recorded 32 cases of poliomyelitis derived from wild poliovirus and 105 cases of poliomyelitis derived from circulating vaccine-derived poliovirus. ${ }^{37}$ Furthermore, in the recent humanitarian crisis due to the civil war in Syria, the WHO officially acknowledged an outbreak on October 29, 2013. In May 2014, the WHO declared polio to be a global health emergency for the first time in the organization's history. This was a substantial challenge to the 25-year-old efforts of the Global Polio Eradication Initiative, which had been successful in eliminating polio from Syria in $1995 .^{38}$ Specifically in Brazil, the authorities reported difficulties in attaining vaccine coverage in the 2018 campaign against paralytic poliomyelitis. ${ }^{39}$ For this reason, the Brazilian authorities have launched another vaccine campaign in order to reach the vaccine coverage recommended by the WHO. ${ }^{39}$ Altogether, this information highlights the need to improve knowledge about poliomyelitis and PPS among healthcare professionals.

Our study had some limitations. Firstly, like all studies in which questionnaires are used, the present results rely on the honesty and level of recall of the respondents. Secondly, the reliability and validity of the instrument used to gather the data for this study has not been determined, although the questionnaire was previously evaluated by two experienced researchers. Nevertheless, we believe that these limitations do not prevent us from drawing conclusions from this study.

\section{CONCLUSION}

Our study showed that, overall, there is a lack of knowledge about PPS and poliomyelitis, especially among psychologists and nutritionists. Therefore, the services provided by these professionals may become compromised. Furthermore, government initiatives should be implemented to increase knowledge among healthcare professionals.

\section{REFERENCES}

1. Howard RS. Poliomyelitis and the postpolio syndrome. BMJ. 2005;330(7503):1314-8. PMID: 15933355; https://doi.org/10.1136/ bmj.330.7503.1314.

2. Schmidt S, Gocheva V, Zumbrunn T, et al. Treatment with L-citrulline in patients with post-polio syndrome: study protocol for a singlecenter, randomised, placebo-controlled, double-blind trial. Trials. 2017;18(1):116. PMID: 28274276; https://doi.org/10.1186/s13063017-1829-3.

3. World Health Organization. Poliomyelitis. 2019. Available from: https:// www.who.int/news-room/fact-sheets/detail/poliomyelitis. Accessed in 2021 (Apr 16)

4. Brasil. Ministério da Saúde. Alerta: 312 cidades têm baixa cobertura vacinal da pólio. Available from: https://www.gov.br/saude/pt-br/ assuntos/noticias/ministerio-da-saude-alerta-para-baixas-coberturasvacinais-para-polio. Accessed in 2021 (Apr 16).

5. Dencker A, Sunnerhagen KS, Taft C, Lundgren-Nilsson Å. Multidimensional fatigue inventory and post-polio syndrome - a Rasch analysis. Health Qual Life Outcomes. 2015;13:20. PMID: 25879413; https://doi. org/10.1186/s12955-015-0213-9. 
6. Halstead LS, Rossi CD. New problems in old polio patients: results of a survey of 539 polio survivors. Orthopedics. 1985;8(7):845-50. PMID: 3867865.

7. Garip Y, Eser F, Bodur H, et al. Health related quality of life in Turkish polio survivors: Impact of post-polio on the health related quality of life in terms of functional status, severity of pain, fatigue, and social, and emotional functioning. Rev Bras Reumatol. 2017;57(1):1-7. PMID: 28137397; https://doi.org/10.1016/j.rbre.2014.12.006.

8. de Lira CAB, de Almeida Alves TM, Peixinho-Pena LF, et al. Knowledge among physical education professionals about poliomyelitis and postpoliomyelitis syndrome: a cross-sectional study in Brazil. Degener Neurol Neuromuscul Dis. 2013;3:41-6. PMID: 30890893; https://doi. org/10.2147/DNND.S45980.

9. Lo JK, Robinson LR. Postpolio syndrome and the late effects of poliomyelitis. Part 1. pathogenesis, biomechanical considerations, diagnosis, and investigations. Muscle Nerve. 2018;58(6):751-9. PMID: 29752819; https://doi.org/10.1002/mus.26168.

10. Koopman FS, Beelen A, Gerrits KH, et al. Exercise therapy and cognitive behavioural therapy to improve fatigue, daily activity performance and quality of life in Postpoliomyelitis Syndrome: the protocol of the FACTS-2-PPS trial. BMC Neurol. 2010;10:8. PMID: 20082714; https://doi. org/10.1186/1471-2377-10-8.

11. Romigi A, Pierantozzi M, Placidi F, et al. Restless legs syndrome and post polio syndrome: a case-control study. Eur J Neurol. 2015;22(3):472-8. PMID: 25393503; https://doi.org/10.1111/ene.12593.

12. Romigi A, Pierantozzi M, Izzi F, et al. Restless legs syndrome and poliomyelitis: new evidences of an old observation? Front Neurol. 2015;6:23. PMID: 25713558; https://doi.og/10.3389/fneur.2015.00023.

13. Sunnerhagen KS, Lundgren-Nilsson A, Willén C. Functioning of the upper extremity in persons with late polio. Eur J Neurol. 2011;18(2):3548. PMID: 20629721; https://doi.org/10.1111/j.1468-1331.2010.03156.x.

14. Voorn EL, Gerrits KH, Koopman FS, Nollet F, Beelen A. Determining the Anaerobic Threshold in Postpolio Syndrome: Comparison With Current Guidelines for Training Intensity Prescription. Arch Phys Med Rehabil. 2014;95(5):935-40. PMID: 24491465; https://doi.org/10.1016/j. apmr.2014.01.015.

15. Voorn EL, Brehm MA, Beelen A, et al. Reliability of Contractile Properties of the Knee Extensor Muscles in Individuals with Post-Polio Syndrome. PLoS One. 2014;9(7):e101660. PMID: 25019943; https://doi.org/10.1371/ journal.pone.0101660.

16. Winberg C, Carlsson G, Brogårdh C, Lexell J. The Perception of Physical Activity in Ambulatory Persons with Late Effects of Polio: A Qualitative Study. J Aging Phys Act. 2017;25(1):65-72. PMID: 27337739; https://doi. org/10.1123/japa.2015-0282.

17. Wiechers DO, Hubbell SL. Late changes in the motor unit after acute poliomyelitis. Muscle Nerve. 1981;4(6):524-8. PMID: 6273721; https:// doi.org/10.1002/mus.880040610.

18. Koopman FS, Beelen A, Gilhus NE, de Visser M, Nollet F. Treatment for postpolio syndrome. Cochrane Database Syst Rev. 2015;18(5):CD007818. PMID: 25984923; https://doi.org/10.1002/14651858.CD007818.pub3.
19. Kosaka T, Kuroha Y, Tada M, et al. A fatal neuromuscular disease in an adult patient after poliomyelitis in early childhood: Consideration of the pathology of post-polio syndrome. Neuropathology. 2013;33(1):93-101. PMID: 22672529; https://doi.org/10.1111/j.1440-1789.2012.01327.x.

20. Tersteeg IM, Koopman FS, Stolwijk-Swüste JM, Beelen A, Nollet F. A 5-Year Longitudinal Study of Fatigue in Patients With Late-Onset Sequelae of Poliomyelitis. Arch Phys Med Rehabil. 2011;92(6):899-904. PMID: 21621666; https://doi.org/10.1016/j.apmr.2011.01.005.

21. Dalakas MC. The post-polio syndrome as an evolved clinical entity. Definition and clinical description. Ann N Y Acad Sci. 1995;753:68-80. PMID: 7611661; https://doi.org/10.1111/j.1749-6632.1995.tb27532.x.

22. de Lira CAB, Vancini RL, Cabral FR, et al. Post-polio syndrome: renaissance of poliomyelitis? Einstein. 2009;7(2 Pt 1)225-8. Available from: http:// apps.einstein.br/revista/arquivos/PDF/1236-Einsteinv7n2p225-8_ing. pdf. Accessed in 2021 (Apr 16).

23. Willén C, Thorén-Jönsson AL, Grimby G, Sunnerhagen KS. Disability in a 4-year follow-up study of people with post-polio syndrome. J Rehabil Med. 2007;39(2):175-80. PMID: 17351702; https://doi. org/10.2340/16501977-0034.

24. Nathanson N, Kew OM. From emergence to eradication: the epidemiology of poliomyelitis deconstructed. Am J Epidemiol. 2010;172(11):1213-29. PMID: 20978089; https://doi.org/10.1093/ aje/kwq320.

25. Lo JK, Robinson LR. Post-polio syndrome and the late effects of poliomyelitis: Part 2. treatment, management, and prognosis. Muscle Nerve. 2018;58(6):760-9. PMID: 29752826; https://doi.org/10.1002/ mus.26167.

26. Vancini RL, Lira CA, Gomes da Silva S, et al. Evaluation of physical educators' knowledge about epilepsy. Arq Neuropsiquiatr. 2010;68(3):367-71. PMID: 20602037; https://doi.org/10.1590/S0004-282X2010000300007.

27. Vancini RL, Benedito-Silva AA, Sousa BS, et al. Knowledge about epilepsy among health professionals: a cross-sectional survey in Sao Paulo, Brazil. BMJ Open. 2012;2(2):e000919. PMID: 22517981; https://doi.org/10.1136/ bmjopen-2012-000919.

28. Boyle M, Sitler M, Kimura I, Rogers K, Duffy AJ. Knowledge and Attitudes of Certified Athletic Trainers in Pennsylvania Toward HIV/AIDS and Treating HIV-Positive Athletes. J Athl Train. 1997;32(1):40-4. PMID: 16558431.

29. Zientek CE, Dewald LL. Cancer detection: the educational role of the athletic trainer. J Athl Train. 1998;33(1):45-9. PMID: 16558484.

30. Strategies for Improving Diabetes Care in Nigeria (SIDCAIN) Research Group. Knowledge of diabetes and hypertension care among health care workers in southwest Nigeria. Postgrad Med. 2009;121(1):173-7. PMID: 19179829; https://doi.org/10.3810/pgm.2009.01.1965.

31. Kelley K, Clark B, Brown V, Sitzia J. Good practice in the conduct and reporting of survey research. Int J Qual Heal Care. 2003;15(3):261-6. PMID: 12803354; https://doi.org/10.1093/intghc/mzg031.

32. Burns KEA, Duffett $M$, Kho ME, et al. A guide for the design and conduct of self-administered surveys of clinicians. Can Med Assoc J. 2008;179(3):24552. PMID: 18663204; https://doi.org/10.1503/cmaj.080372. 
33. Morin CA, Roberts CL, Mshar PA, Addiss DG, Hadler JL. What do physicians know about cryptosporidiosis? A survey of Connecticut physicians. Arch Intern Med. 1997;157(9):1017-22. PMID: 9140274; https://doi. org/10.1001/archinte.1997.00440300137012.

34. Matyja E. Zespół post-polio. Część Il. Postępowanie terapeutyczne [Postpolio syndrome. Part II. Therapeutic management]. Neurol Neurochir Pol. 2012;46(4):372-8. PMID: 23023436; https://doi.org/10.5114/ ninp.2012.30270.

35. Laurenti R, Nubila HB, Quadros AA, Conde MT, Oliveira AS. A Classificação Internacional de Doenças, a Família de Classificações Internacionais, a CID-11 e a Síndrome Pós-Poliomielite [The International Classification of Diseases, the Family of International Classifications, the ICD-11, and post-polio syndrome]. Arq Neuropsiquiatr. 2013;71(9A):3-10. PMID: 24141431; https://doi.org/10.1590/0004-282X20130111.

36. Trojan DA, Cashman NR, Shapiro S, Tansey CM, Esdaile JM. Predictive factors for post-poliomyelitis syndrome. Arch Phys Med Rehabil. 1994;75(7):770-7. PMID: 8024423.

37. World Health Organization. Global Polio Eradication Initiative (GPEI). Polio Now. GPEl. Geneva:WHO; 2019. Available from: http://polioeradication. org/polio-today/polio-now/. Accessed in 2021 (Apr 16).

38. Al-Moujahed A, Alahdab F, Abolaban H, Beletsky L. Polio in Syria: Problem still not solved. Avicenna J Med. 2017;7(2):64-6. PMID: 28469988; https:// doi.org/10.4103/ajm.AJM_173_16.

39. Brasil. Ministério da Saúde. Começa na segunda vacinação contra poliomielite e sarampo. Ministério da Saúde; 2018. Available from: https:// www-hmg.saude.gov.br/noticias/agencia-saude/43976-segunda-e-diade-vacina-contra-polio-e-sarampo. Accessed in 2021 (Apr 16).

Acknowledgements: We would like to thank all of the participants who volunteered their time to participate in the study

Availability of data and materials: The datasets used and/or analyzed during the current study are available from the corresponding author upon reasonable request

Authors' contributions: de Lira CAB: conceptualization (equal), data curation (equal), formal analysis (equal), investigation (equal), methodology (equal) and writing-review and editing (equal); Santos DAT: conceptualization (equal), formal analysis (equal), methodology (equal) and writing-original draft (equal); Viana RB: conceptualization (equal), investigation (equal), methodology (equal) and writing-original draft (equal); Guimarães JM: conceptualization (equal), investigation (equal), methodology (equal) and writing-original draft (equal); Oliveira JNS: conceptualization (equal), investigation (equal), methodology (equal) and writing-original draft (equal); Sousa BS: conceptualization (equal), formal analysis (equal), investigation (equal) and writingoriginal draft (equal); Santana MG: conceptualization (equal), data curation (equal), project administration (equal) and writing-review and editing (equal); Vancini RL: conceptualization (equal), investigation (equal), project administration (equal) and writing-review and editing (equal); Andrade MS: conceptualization (equal), investigation (equal), project administration (equal) and writing-review and editing (equal); Nikolaidis P: conceptualization (equal), project administration (equal), supervision (equal) and writing-review and editing (equal); Rosemann T: conceptualization (equal), project administration (equal), supervision (equal) and writing-review and editing (equal); Knechtle B: conceptualization (equal), project administration (equal), supervision (equal) and writing-review and editing (equal). All authors actively contributed to discussion of the study results, and reviewed and approved the final version to be released

Sources of funding: This work was supported by the Fundação de Amparo à Pesquisa do Estado de Goiás (FAPEG-Brazil) (grant number 201210267001056) and financed in part by the Coordenação de Aperfeiçoamento de Pessoal de Nível Superior - Brasil (CAPES), under finance code 001. DATS had a fellowship from the Programa de Apoio à Capacitação Docente e de Técnicos Administrativos da Universidade do Estado da Bahia (PAC-DT/UNEB, Brazil) and RBV had a fellowship from Coordenação de Aperfeiçoamento de Pessoal de Ensino Superior (CAPES, Brazil)

Conflict of interest: The authors declare that they have no competing interests

Date of first submission: October 15, 2020

Last received: October 15, 2020

Accepted: March 16, 2021

\section{Address for correspondence:}

Claudio Andre Barbosa de Lira

Faculdade de Educação Física e Dança, Universidade Federal de Goiás (UFG)

Av. Esperança, s/no

Campus Samambaia — Goiânia (GO), Brasil

CEP 74690-900

Tel. Fax. (+55 62) 3521-1141

E-mail: andre.claudio@gmail.com 